\title{
Políticas públicas e direitos dos usuários do Sistema Único de Saúde com diabetes mellitus
}

\author{
Public policy and rights of Single Health System's users with diabetes mellitus
}

Las políticas públicas y los derechos de los usuarios del Sistema Único de Salud con diabetes mellitus

\section{Ellen Cristina Barbosa dos Santos', Carla Regina de Souza Teixeira", Maria Lúcia Zanetti"',, Manoel Antônio dos Santosiv ${ }^{\mathrm{IV}}$, Marta Cristiane Alves Pereira ${ }^{\mathrm{V}}$}

\author{
' Universidade de São Paulo, Escola de Enfermagem de Ribeirão Preto, Departamento de Enfermagem Geral e \\ Especializada, Programa de Pós-Graduação em Enfermagem Fundamental. (Mestranda) Ribeirão Preto-SP, Brasil. \\ " Universidade de São Paulo, Escola de Enfermagem de Ribeirão Preto. Centro Colaborador \\ da OMS para o Desenvolvimento da Pesquisa em Enfermagem. Ribeirão Preto-SP, Brasil. \\ "' Universidade de São Paulo, Escola de Enfermagem de Ribeirão Preto, Departamento de Enfermagem Geral e \\ Especializada. Centro Colaborador da OMS para o Desenvolvimento da Pesquisa em Enfermagem. \\ Ribeirão Preto-SP, Brasil. \\ "v Universidade de São Paulo, Faculdade de Filosofia, Ciências e Letras de Ribeirão Preto, \\ Departamento de Psicologia e Educação. Ribeirão Preto-SP, Brasil. \\ $\checkmark$ Universidade de São Paulo, Escola de Enfermagem de Ribeirão Preto, Departamento de Enfermagem Geral e \\ Especializada, Programa de Pós-Graduação em Enfermagem Fundamental. (Doutoranda) Ribeirão Preto-SP, Brasil.
}

Submissão: 04-08-2009 Aprovação: 05-09-2011

\section{RESUMO}

Este estudo aborda a relação entre políticas públicas e direitos dos usuários com diabetes mellitus quanto aos insumos utilizados no tratamento da doença, sob o ponto de vista das normas e procedimentos legais de amparo a essa população. Preconizase que os profissionais de saúde devem constituir um elo entre os usuários e o atual modelo de saúde vigente, assumindo ativamente um papel de defesa dos direitos dos usuários com diabetes mellitus do sistema único de saúde. Essa postura é um ideal a ser conquistado e consolidado no movimento das práticas cotidianas. Para tanto, é preciso fortalecer o processo educativo, incluindo a dimensão ético-político durante as ações implementadas com os usuários do sistema de saúde.

Descritores: Diabetes mellitus, direitos do paciente, políticas públicas.

\begin{abstract}
This study approaches the relationship between public policies and the rights of SUS (Single Health System) users with diabetes mellitus regarding the supplies used in the treatment of diabetes, under the view of legal rules and procedures for support of this population. Health professionals should be a link between users and current health model, actively assuming a role of defending the rights of SUS users with diabetes mellitus. This posture is an ideal to be achieved and consolidated in daily practices. The educational process needs to be strengthened, including the ethical-political dimension during actions implemented with SUS users.
\end{abstract}

Key words: Diabetes mellitus, Patient rights, Public policies.

\section{RESUMEN}

Este estudio enfoca la relación entre las políticas públicas y los derechos de los usuarios del Sistema Único de Salud (SUS) con diabetes mellitus cuanto a los insumos utilizados en el tratamiento de la enfermedad, bajo el punto de vista de las normas y procedimientos legales de amparo a esa población. Los profesionales de salud deben constituir un eslabón entre los usuarios y el modelo actual de salud vigente, asumiendo activamente un papel de defensa de los derechos de los usuarios del SUS con diabetes. Esa postura es un ideal a ser conquistado y consolidado las prácticas cotidianas. Es necesario fortalecer el proceso educativo, incluyendo la dimensión ético-política durante las acciones implementadas con los usuarios del SUS.

Palabras clave: Diabetes mellitus, Derechos del paciente, Políticas públicas. 


\section{INTRODUÇÃO}

O Diabetes mellitus (DM) é uma condição crônica de saúde que exige cuidados permanentes para seu controle. Esses cuidados estão relacionados aos aspectos nutricionais, ao incremento da atividade física regular, uso de terapêutica medicamentosa e auto monitorização da glicemia capilar no domicílio, entre outros. Para instituir e manter esses cuidados de modo sistemático é necessário o apoio de uma equipe multiprofissional capacitada e insumos para favorecer um meIhor controle metabólico(1).

Para o alcance de um bom controle metabólico, a pessoa com DM necessita introduzir mudanças nos hábitos de vida. Essa é uma tarefa difícil e deve levar em consideração alguns fatores que se encontram intimamente entrelaçados com as questões instrumentais e comportamentais. Tais fatores reportam-se a um conjunto de influências interpessoais familiares e de pares, e que se encontram alicerçados em crenças e valores que interferem na motivação e na capacidade da pessoa enfrentar a sua doença e buscar soluções para controlá-la. Assim, é importante entender os padrões individuais de resposta da pessoa com diabetes, bem como de sua família em relação aos seus sentimentos, angústias, ansiedades, conflitos e necessidades ${ }^{(2-3)}$.

O tratamento da pessoa com diabetes tem como finalidade obter um adequado controle metabólico e deve abranger um programa de educação continuada, modificações no estilo de vida, que incluem aumento da atividade física, reorganização dos hábitos alimentares, abolição do tabagismo e, se necessário, uso de medicamentos ${ }^{(1,3)}$. No entanto, as dificuldades do usuário em utilizar a medicação prescrita, seguir rigorosamente o planejamento alimentar recomendado ou modificar seu estilo de vida, de acordo com as orientações da equipe multiprofissional, é um problema sempre presente na prática clínica ${ }^{(4)}$.

Tendo em vista a complexidade da doença e do tratamento e de acordo com o que se pode observar na prática clínica, o DM é considerado um problema de importância crescente em saúde pública ${ }^{(5-6)}$. Além de afetar a produtividade, a qualidade de vida e a sobrevida das pessoas, o DM envolve altos custos para manutenção do tratamento e prevenção e/ou tratamento de complicações crônicas e agudas ${ }^{(7)}$.

Segundo um estudo realizado com o objetivo de descrever as hospitalizações por DM no Brasil, no período de 1999 e 2001, foi possível observar que o número de internações por DM é elevado e possui diferenças regionais ${ }^{(8)}$. Dessa forma, os custos em saúde aumentam e se encontram relacionados à alta taxa de permanência hospitalar da pessoa com diabetes e também à gravidade das complicações, que muitas vezes demandam procedimentos de alta complexidade.

Torna-se evidente, também, a necessidade de empenho de lideranças do governo e gestores de saúde na criação de mecanismos que minimizem o aparecimento, bem como o agravamento da doença ${ }^{(9)}$. Esses esforços devem ser direcionados tanto à reorganização da atenção à pessoa com diabetes quanto ao provimento de insumos necessários para o controle da doença, com o intuito de reduzir os custos advindos da não detecção precoce e das complicações relacionadas à falta de controle metabólico no $\mathrm{DM}^{(10)}$.
Dada a necessidade de proporcionar um serviço de atenção à pessoa com diabetes de elevada qualidade e de racionalizar custos, políticas públicas têm sido instituídas no contexto brasileiro. Dentre as políticas de saúde, o fornecimento de insumos para melhor acompanhamento das pessoas acometidas pelo DM é uma estratégia que está em fase de consolidação pelo SUS.

Dessa forma, objetiva-se com este artigo, realizar uma reflexão sobre a relação entre políticas públicas e os direitos dos usuários com diabetes mellitus, sob o ponto de vista das normas e procedimentos legais de amparo a essa população. Ademais, busca-se também aprofundar a reflexão no sentido de conhecer as políticas de apoio aos usuários com diabetes e reconhecer quais seriam as barreiras para a real consolidação da legislação de proteção e amparo aos direitos dos usuários do sistema único de saúde, em especial os usuários com DM.

\section{REFLEXÃO E DISCUSSÃO}

Políticas públicas de facilitação do acesso da pessoa com diabetes aos insumos e medicamentos para o tratamento

Tendo em vista a crescente necessidade de formular políticas capazes de suprir as demandas em doenças crônicas, sem elevar os custos em saúde, o governo do Estado de São Paulo, em março de 2001, promulgou a lei no 10.782 que definiu as diretrizes para uma política de prevenção e atenção integral a saúde da pessoa com diabetes mellitus, no âmbito do SUS ${ }^{(11)}$.

Em 2002, o Ministério da Saúde, em conjunto com sociedades científicas, elaboraram o Plano de Reorganização da Atenção à Hipertensão Arterial e ao Diabetes Mellitus ${ }^{(12)}$. Esse plano teve como objetivo reduzir: o número de internações, a procura pelo pronto-atendimento nas Unidades Básicas de Saúde, os gastos com tratamento de complicações crônicas, aposentadorias precoces e mortalidade cardiovascular, de modo a promover melhoria da qualidade de vida da população.

Com base nos direitos advindos da Carta dos Direitos dos Usuários da Saúde, sobretudo o direito ao tratamento adequado e efetivo para o seu problema, e dos acordos entre as três esferas nacionais, em 29 de setembro de 2007, entrou em vigor a Lei Federal $n^{\circ} 11.347 / 06^{(13)}$. Essa dispõe sobre a distribuição gratuita de medicamentos, materiais para aplicação de insulina e monitorização da glicemia capilar. Tal distribuição está condicionada ao cadastramento dos usuários nas unidades de saúde de sua área de abrangência e nos programas de educação em diabetes.

É preciso destacar que, em Ribeirão Preto, como também outras cidades brasileiras, a Secretaria Municipal de Saúde já havia implantado, em agosto de 2005, a lei $n^{\circ} 10.299$, que dispõe sobre as normas e procedimentos de proteção e amparo ao usuário com diabetes ${ }^{(14)}$. Nessa lei estão garantidos os direitos dos usuários com DM a medicamentos, instrumentos para autoaplicação de insulina e automonitoração da glicemia capilar no domicílio. Desse modo, foram antecipados, no âmbito da saúde pública municipal, os direitos que seriam reconhecidos pela Legislação Federal somente em 2007.

Assim, a Secretaria Municipal de Saúde de Ribeirão Preto, em conjunto com a Secretaria de Saúde do Estado de São 
Paulo, disponibilizam, gratuitamente, glicosímetro, lancetador, fitas reagentes e lancetas para usuários de insulina, desde agosto de $2005^{(14)}$. Contudo, apesar dos esforços envidados, ainda percebe-se que o esquema ideal de monitoração de glicemia para todos os usuários com diabetes ainda é impraticável do ponto de vista econômico, o que inviabiliza o fornecimento dos glicosímetros e insumos para todos os usuários do SUS com DM. Essa limitação é incompatível com as legislações nos âmbitos federal, estadual e municipal, que preconizam a atenção integral ao usuário com diabetes mellitus, bem como o direito a medicamentos e insumos necessários para o controle da doença ${ }^{(15)}$.

Faz-se necessário salientar que, mesmo depois da reforma sanitária que implantou o SUS em 1990, a distribuição gratuita dos medicamentos aos usuários com diabetes mellitus só foi regulamentada em 2007, ou seja, 17 anos após sua proposição, o que demonstra as inúmeras dificuldades ainda existentes para a real efetivação dos princípios e diretrizes preconizados.

Reconhece-se que houve avanço no que diz respeito à criação de leis e normas que assegurem o acesso do usuário aos serviços de saúde. Por outro lado, a operacionalização das leis ainda esbarra na falta de mecanismos que sensibilizem os gestores e profissionais de saúde em relação às suas responsabilidades e conscientizem a população sobre os direitos que Ihe são assegurados por lei.

Barreiras para operacionalização da legislação de proteção e amparo ao usuário do sistema único de saúde

Os gestores públicos se deparam com uma série de barreiras e deficiências no que se refere à atenção ao usuário com diabetes mellitus na rede pública de saúde no Brasil. Somente a promulgação da lei de proteção e amparo ao usuário com DM não garante a regulamentação e disponibilização dos recursos necessários para o tratamento da doença, tais como instrumentos e materiais para verificação da glicemia capilar no domicílio, medicamentos e insumos, de forma regular e equitativa para todos, bem como não assegura também a capacitação da equipe multiprofissional de saúde para o atendimento ${ }^{(16-17)}$.

Em consequência do não cumprimento da lei tem-se verificado a crescente utilização de recursos jurídicos, por parte dos usuários, como meio de se obter o acesso aos medicamentos ou insumos necessários aos seus tratamentos ${ }^{(18)}$. Torna-se evidente dessa forma que não basta a formulação e divulgação de documentos que garantam o direito à saúde, mas é necessário assegurá-lo como um direito efetivo de fato ${ }^{(19-20)}$. Em diabetes, os medicamentos que sofrem comumente processos de judicialização são as insulinas sem pico de ação.

O aumento da demanda de processos jurídicos relacionados aos direitos à saúde e a pressão da sociedade civil organizada culminam na elaboração de projetos de leis. O Poder Legislativo tem no dispositivo do projeto de lei o principal instrumento de resposta às demandas sociais ${ }^{(18)}$.

No Brasil são inúmeros os casos de medicamentos que passam a ser fornecidos regularmente por força de decisão judicial, uma vez que não constam da lista oficial do Ministério da Saúde ${ }^{(16)}$. Dados do Ministério da Saúde apontam que, no Estado de São Paulo, as determinações judiciais para fornecimento de medicamentos e tratamentos específicos passaram de 80 para 600 solicitações mensais, perfazendo um aumento de $650 \%$ no intervalo de seis anos. As ações judiciais encontram-se respaldadas no princípio do direito à atenção integral sem limitações e é aplicável a qualquer tratamento que um usuário necessite e que não tenha provisão pelo Estado(18).

Nesse contexto, o SUS enfrenta um paradoxo entre as diferentes demandas de saúde e o conflito ético que diz respeito à alocação dos recursos em saúde. Se, por um lado, os usuários requerem por meios judiciais o acesso a medicamentos de alto custo, por outro, a Rede Básica de Saúde ainda enfrenta dificuldades para oferecer o acesso universal e cuidado integral ao atendimento preconizado pelo Ministério da Saúde. Acresce-se a isso que às vezes também ocorrem problemas relacionados à regularidade no fornecimento de medicamentos já preconizados pelos programas de saúde.

Diante das considerações acima mencionadas, um estudo faz três apontamentos: o primeiro refere-se à importância do reconhecimento das iniciativas do Poder Legislativo como termômetro das pressões sociais, seja no setor saúde ou em qualquer outra área. Esses dados mostram, no caso da saúde, a forte preocupação da sociedade com a extensão da cobertura das prestações do SUS, em particular quanto aos medicamentos $^{(18)}$.

O segundo apontamento diz respeito à baixa eficácia direta desse tipo de ação legislativa, uma vez que o caminho entre a apresentação da proposta e a sanção presidencial é, sabidamente, longo, tortuoso e, via de regra, lento. Contudo, o projeto de lei cumpre um papel importante como fator de mobilização política e social face à pressão que a sociedade, ou parte dela, exerce sobre o Executivo ${ }^{(18)}$.

Por fim, um terceiro apontamento quanto aos motivos da necessidade de se recorrer ao Poder Legislativo, aparentemente redundante, face que a integralidade é um princípio constitucional que dispensaria leis que individualizam cada doença, tratamento ou medicamento. Cabe destacar, ainda, que o princípio da integralidade ainda não foi regulamentado por lei ${ }^{(18)}$.

A propósito, uma das recomendações de um estudo realizado pelo Ministério da Saúde ${ }^{(16)}$, com o objetivo de analisar o financiamento, em âmbito federal, e o acesso aos serviços de maior custo providos pelo SUS, é que os princípios da universalidade e da integralidade sejam regulamentados por lei, uma vez que não podem ser entendidos como tudo para todos no contexto de um sistema de livre demanda. É preciso criar regras claras e transparentes dentro do espírito democrático, sem descuidar do sentido social e equitativo ${ }^{(18)}$.

Desse modo, pode-se detectar que a adoção de novas maneiras de estruturar o sistema de saúde introduz no seu bojo problemas que exigem uma abordagem ao mesmo tempo crítica e de busca de soluções criativas. O modelo de saúde vigente demanda a aquisição de novas competências pelos profissionais, além de novas informações que acompanhem a perspectiva dinâmica do processo saúde-doença, que tenham potencial adaptativo e que respeitem, sobretudo, a ética, o direito e a autonomia dos usuários ${ }^{(21)}$. 
Deve-se levar em conta que o alcance de novas competências não podem se restringir à dimensão técnico-instrumental, tornando-se uma simples estratégia de adaptação às novas necessidades do processo produtivo, mas devem ser consideradas de forma ampliada no sentido de abranger a dimensão ética e política no mundo do trabalho, uma vez que as questões relacionadas à cidadania e aos direitos dos usuários da saúde são repletos de situações e conflitos bioéticos ${ }^{(22)}$.

O conhecimento dos direitos dos usuários é essencial para o desenvolvimento de uma consciência democrática, responsável e reflexiva do cidadão, tanto no papel de usuário quanto no de profissional da saúde ${ }^{(23)}$. Conhecer os direitos é o primeiro passo para diminuir as barreiras enfrentadas pelos usuários com diabetes mellitus para o acesso pleno a seus direitos de cidadania.

\section{Os direitos dos usuários com diabetes mellitus}

A partir dos anos 1980, a bioética começou a ampliar seu foco de interesse, abarcando a clínica em relação ao contexto do sistema de saúde e, consequentemente, incorporou reflexões acerca das questões relativas à estrutura, à gestão e ao financiamento do sistema ${ }^{(21,24)}$. Nesse prisma, entende-se que a Bioética é parte do cotidiano de todo ser humano e, portanto, deve ser enfatizada e voltar-se para os conflitos e dilemas do dia a dia, como os direitos do usuário e o conteúdo do conceito de cidadania em saúde ${ }^{(25)}$.

Uma concepção contemporânea que vem sendo utilizada em bioética é a de que o exercício dos direitos, pelos usuários, deve visar o alcance da humanização dos serviços de saúde, além de garantir a cidadania em saúde. Nesse contexto, o direito à saúde passa a constituir o "valor-mor" do paradigma bioético ${ }^{(24,26)}$. O direito é um componente central da noção de cidadania. Assim, o respeito aos direitos do cidadão, como usuários, constitui um dos principais indicadores de qualidade para o planejamento e gestão dos serviços de saúde $^{(27)}$. Cabe destacar que, no regime democrático, a justiça distributiva refere-se à distribuição dos direitos e responsabilidades na sociedade, incluindo os direitos civis e políticos ${ }^{(21)}$.

Apesar dos avanços conquistados por meio de uma legislação arrojada, constata-se que os usuários de saúde com diabetes, ainda hoje, têm pouco acesso a esses benefícios, exercendo minimamente seus direitos como cidadãos. O processo histórico de construção da cidadania esbarrou em obstáculos de toda natureza, como a segmentação dos direitos por camadas sociais, etnia e sexo, o estímulo ao corporativismo extremado na luta pela preservação dos interesses de cada categoria, os períodos de regime ditatorial em períodos-chave de transformação política e social, a conservação dos grupos dominantes no poder ${ }^{(28)}$.

As inúmeras barreiras para a consolidação dos princípios preconizados pelo SUS podem e deve ser enfrentadas nos embates cotidianos, o que exige a determinação dos gestores e a efetivação de iniciativas que ampliem a mobilização social e promovam a cidadania, favorecendo o acesso e garantindo maior qualidade aos serviços de saúde ${ }^{(29)}$.

Portanto, não se trata apenas da construção de um sistema de normas, portarias ou leis, mas da criação de condições objetivas para a implementação dos dispositivos que garantam o exercício dos direitos na vida cotidiana das pessoas ${ }^{(20)}$. Só assim são asseguradas as condições objetivas para uma efetiva melhora do controle metabólico dos usuários, priorizando a singularidade do ser humano, de modo a propiciar a formação de cidadãos capazes de exercer seus direitos e autonomia, em um quadro de justiça distributiva.

Para ter sentido transformador, as ações de saúde precisam respaldar-se em dois pilares: o primeiro é a garantia de acesso às informações sobre os direitos legais como cidadãos, que deverão ser fornecidas por equipe multiprofissional de saúde capacitada. O segundo, e não menos importante pilar, diz respeito à provisão de recursos, bem como a sua adequada alocação e otimização, para que o acesso seja alcançado de forma justa e equitativa.

Em síntese, duas questões mostram-se pertinentes. A primeira concerne ao conhecimento que os usuários com diabetes possuem acerca de seus direitos e a segunda está relacionada ao paradoxo existente entre as inúmeras tecnologias disponíveis na atualidade e a restrição do acesso aos insumos.

No que diz respeito à utilização adequada dos materiais e às condutas a serem tomadas frente aos resultados obtidos nos exames de glicemia capilar realizadas no domicílio ainda se observam nos serviços de saúde deficiências no acolhimento, relacionadas às informações necessárias para o manejo da doença. Esse fator constitui uma barreira para o estabelecimento de uma comunicação eficaz entre profissionais de saúde e usuários, prejudicando a formação do vínculo de confiança, que é um requisito primordial para um trabalho colaborativo ${ }^{(30)}$.

Em pesquisa realizada em serviços de atenção básica nos Estados Unidos da América com diversos profissionais de saúde - médicos, enfermeiros, fisioterapeutas, entre outros confirmou-se que ocorrências éticas comuns na atenção básica configuram as preocupações pragmáticas do dia a dia, especialmente as ligadas à prática clínica ${ }^{(31)}$. No referido estudo, 12 problemas foram apontados como os mais freqüentes e ocorrem reiteradamente no cotidiano dos serviços de saúde, dentre eles temos: a falta de preparo e atualização dos profissionais de saúde, o trato desrespeitoso e a informação inadequada dispensada aos usuários, dentre outros.

Nesse contexto produzido pela legitimação dos direitos dos usuários com DM, a bioética atua como instância moderadora das ações de saúde e de suas repercussões na vida das pessoas, ao conduzir à elaboração de reflexões sobre o acesso aos direitos dos usuários, bem como a disseminação de informações relativas ao reconhecimento e provimento desses direitos pelos serviços de saúde.

\section{CONSIDERAÇÕES FINAIS}

Para que os direitos do usuário com DM, previstos em lei através da política de atenção aos usuários com diabetes, sejam efetivamente implementados, torna-se necessário uma ampla divulgação, de modo a disseminar o conhecimento pela população e pelos profissionais de saúde. Considerando que a equipe multiprofissional em saúde deve ser um elo 
entre os usuários e o atual modelo de saúde vigente, cada vez mais há necessidade não apenas de educar os usuários com o intuito de oferecer informações acerca da doença e seu tratamento, mas também de conscientizá-los de seus direitos de cidadania, assumindo um papel de defesa de suas prerrogativas. É preciso, portanto, fortalecer o processo educativo, incluindo a dimensão ética-política durante as ações implementadas com os usuários do sistema de saúde.

Essa postura é um ideal a ser conquistado e consolidado no movimento das práticas cotidianas. Para tanto, é indispensável uma mudança de paradigma. Os profissionais de saúde devem dispor de competências do ponto de vista científico, tecnológico e bioético para exercerem suas funções em diferentes contextos sociais. Dessa maneira, é crescente a responsabilidade dos profissionais de saúde, como seres pró-ativos, de incorporarem uma postura reflexiva e ética que contemple a adoção de novos conhecimentos e habilidades necessários para a efetiva comunicação com os usuários com diabetes, configurando um ambiente propício para que as ações de saúde sejam traduzidas em um cuidado humanizado.

\section{REFERÊNCIAS}

1. Arauz AG, Sánchez G, Padilha G, Fernández M, Rosello M, Guzmán S. Intervención educativa comunitária sobre la diabetes en el ámbito de la atención primária. Rev. Panam. Salud Pública 2001;9 (3):145-53.

2. Santos ECB, Zanetti ML, Otero LM, Santos MA. O cuidado sob a ótica do paciente diabético e seu principal cuidador. Rev. Latino-Am. Enfermagem 2005;13(3):397-406.

3. Silva DGV, Souza SS, Francioni FF, Mattosinho MMS, Coelho MS, Sandoval RCB, et al. Pessoas com Diabetes mellitus: suas escolhas de cuidados e tratamentos. Rev Bras Enferm. 2006;59(3): 297-302.

4. Faria HTG, Zanetti ML, Santos MA, Teixeira CRS. Conhecimento sobre terapêutica medicamentosa em diabetes: um desafio na atenção à saúde. Acta Paul. Enferm. 2009;22(5):612-7.

5. Barbosa RB, Barceló A, Machado CA. Campanha nacional de detecção de casos suspeitos de diabetes mellitus no Brasil: relatório preliminar. Rev. Panam. Salud Publica 2001;10(5):5-30.

6. Holmstrom IM, Rosenqvist U. Misunderstandings about illness and treatment among patients with type 2 diabetes. J. Adv. Nurs. 2005;49(2):146-54.

7. Davis WA, Knuiman MW, Hendrie DMA, Davis TME. Determinants of diabetes-attributable non-blood glucoselowering medications costs in type 2 diabetes: the Fremantle Diabetes Study. Diabetes Care. 2005;28 (2):329-36.

8. Rosa RS, Schmidt MI, Duncan BB, Souza MFM, Lima AK, Moura L. Internações por Diabetes Mellitus como diagnóstico principal na Rede Pública do Brasil, 1999-2001. Rev. Bras. Epidemiol 2007;10(4):465-78.

9. Organização Mundial da Saúde. Cuidados inovadores para condições crônicas: componentes estruturais de ação: relatório mundial. Brasília (DF): Organização Mundial da Saúde; 2003.

10. Oliveira SLC, Lunardi Filho WD. AIDS e Diabetes mellitus versus justiça distributiva no sistema público de saúde. Rev Bras Enferm 2004;57(6):750-3.

11. São Paulo. Lei $n^{\circ} 10.782$, de 9 de março 2001. Define diretrizes para uma política de prevenção e atenção integral à saúde da pessoa portadora de diabetes, no âmbito do Sistema Único de Saúde e dá outras providências. Diário Oficial da União, Brasília (2001, mar, 09).
12. Ministério da Saúde (BR). Plano de reorganização da atenção à hipertensão arterial e ao diabetes mellitus. Brasília (DF): Ministério da Saúde; 2002.

13. Brasil. Lei $n^{\circ} 11.347$, de 27 de setembro de 2006. Dispõe sobre a distribuição gratuita de medicamentos e materiais necessários à sua aplicação e à monitoração da glicemia capilar aos portadores de diabetes inscritos em programas de educação para diabéticos. Diário Oficial da União, Brasília (2006, set, 28).

14. Ribeirão Preto. Lei $n^{\circ} 10.299$, de 22 de dezembro de 2004. Dispõe sobre as normas de proteção à pessoa diabética incluindo o fornecimento de medicamento e a monitorização da glicemia capilar aos usuários diabéticos cadastrados em programas de educação em diabetes. Diário Oficial, Ribeirão Preto (2004, dez, 22).

15. Castro ARV, Grossi SAA. Custo do tratamento do diabetes mellitus tipo 1: dificuldades das famílias. Acta Paul. Enferm. 2008;21(4):624-8.

16. Assunção MCF, Santos IS, Gigante DP. Atenção primária em diabetes no Sul do Brasil: estrutura, processo e resultado. Rev Saúde Pública 2001;35(1):88-95.

17. Assunção MCF, Santos IS, Costa JSD. Avaliação do processo da atenção médica: adequação do tratamento de pacientes com diabetes mellitus, Pelotas, Rio Grande do Sul, Brasil. Cad Saúde Pública 2002;18(1):205-11.

18. Ministério da Saúde (BR). Atenção de alta complexidade no SUS: desigualdades no acesso e no financiamento. Brasília (DF): Ministério da Saúde; 2005.

19. Dallari SG. O direito à saúde na visão de um conselho municipal de saúde. Cad de Saúde Pública 1996; 12 (4): 531-540.

20. Gomes ILV, Caetano R, Jorge MSB. A criança e seus direitos na família e na sociedade: uma cartografia das leis e resoluções. Rev. Bras, Enferm 2008;61(1):61-5.

21. Zoboli ELCP. Bioética e atenção básica: um estudo de ética descritiva com enfermeiros e médicos do Programa de Saúde da Família. [doutorado]. São Paulo(SP): Faculdade de Saúde Pública da Universidade de São Paulo; 2003.

22. Deluiz N. Qualificação, competências e certificação: visão do mundo do trabalho. Formação $2001 ;(2): 5-17$.

23. Gauderer EC. Os direitos do paciente. Rio de Janeiro: Record; 1998. 
24. Pessini L, Barchifontaine CP. Problemas atuais de bioética. São Paulo: Loyola; 2000.

25. Selli L. Bioética na enfermagem. São Leopoldo (RS): Unisinos; 2003.

26. Garrafa V, Oselka G, Diniz D. Saúde pública, bioética e equidade. Bioética 1997;5(1):27-33.

27. Fortes PAC. Ética, direitos dos usuários e políticas de humanização da atenção à saúde. Saúde soc. 2004;13(3):30-5.

28. Baptista TWF. O direito à saúde no Brasil: sobre como chegamos no Sistema Único de Saúde e o que esperamos dele. In: Venâncio J. Políticas de saúde. Rio de Janeiro: Editora Fiocruz, 2005.
29. Ministério da Saúde (BR). Direitos dos usuários dos serviços e das ações de saúde no Brasil: legislação compilada - 1973 a 2006. Brasília (DF): Ministério da Saúde; 2007.

30. Péres DS, Santos MA, Zanetti ML, Ferronato AA. Dificuldades dos pacientes diabéticos para o controle da doença: sentimentos e comportamentos. Rev. Latino-Am. Enfermagem 2007;15(6):1105-12.

31. Robilard HM, High DM, Sebastian JG, Pisaneschi JI, Perritt LJ, Mahler DM. Ethical issues in primary health care: a survey of practioners' perceptions. J Community Health 1989;14(1):9-17. 